\title{
Use of Simulated Atrophy for Performance Analysis of Brain Atrophy Estimation Approaches ${ }^{\star}$
}

\author{
Swati Sharma ${ }^{1,2}$, Vincent Noblet ${ }^{2}$, François Rousseau ${ }^{2}$, Fabrice Heitz ${ }^{2}$, \\ Lucien Rumbach ${ }^{1,3}$, and Jean-Paul Armspach ${ }^{1}$ \\ 1 LINC-IPB (UMR CNRS-UDS 7191), Strasbourg, France \\ swati.sharma@linc.u-strasbg.fr \\ 2 LSIIT (UMR CNRS-UDS 7005), Strasbourg, France \\ 3 Centre Hospitalier Universitaire, Besançon, France
}

\begin{abstract}
In this paper, we study the performance of popular brain atrophy estimation algorithms using a simulated gold standard. The availability of a gold standard facilitates a sound evaluation of the measures of atrophy estimation, which is otherwise complicated. Firstly, we propose an approach for the construction of a gold standard. It involves the simulation of a realistic brain tissue loss based on the estimation of a topology preserving B-spline based deformation fields. Using this gold standard, we present an evaluation of three standard brain atrophy estimation methods (SIENA, SIENAX and BSI) in the presence of bias field inhomogeneity and noise. The effect of brain lesion load on the measured atrophy is also evaluated. Our experiments demonstrate that SIENA, SIENAX and BSI show a deterioration in their performance in the presence of bias field inhomogeneity and noise. The observed mean absolute errors in the measured Percentage of Brain Volume Change (PBVC) are $0.35 \% \pm 0.38,2.03 \% \pm 1.46$ and $0.91 \% \pm 0.80$ for SIENA, SIENAX and BSI, respectively, for simulated whole brain atrophies in the range $0-1 \%$.
\end{abstract}

\section{Introduction}

The last decade has seen the emergence of sophisticated image processing techniques, based on Magnetic Resonance Imaging (MRI) acquisitions, for assessing the brain volume. The associated ease of use and portability of these methods has attracted the attention of the medical community in a large way. This has led to an increasing interest in the use of the brain atrophy measurements as a reliable index of disease progression since brain atrophy is a common feature of many neuro-degenerative diseases such as Multiple Sclerosis (MS) 1], Alzheimer's disease (AD) and Dementia. Although, the existing approaches for

\footnotetext{
* We are thankful to Alsace Region and ARSEP for supporting this study. We also thank Dr. Evan Fletcher (University of California, Davis) for his support on the BSI software.
} 
measuring atrophy are sensitive and reproducible, their accuracy and reliability are affected by a number of factors. For instance, non-destructive biologic factors such as inflammation, edema, steroid therapy, dehydration, alcohol consumption and normal aging may contribute to a change in the brain volume. MRI artefacts including motion artefacts, sequence variations, bias field inhomogeneity, noise and others may influence the brain atrophy measurements. Other sources of error are more method specific and include inaccuracies in registration and segmentation of images. Since these tools are being increasingly used as a marker of disease evolution in many pathologies, their validation is a key problem. However, in the real scenario, the non-availability of the ground truth complicates the evaluation and comparison of these techniques. In this paper, we address the problem of creation of a gold standard. We propose a topology preserving non-rigid registration based framework for simulating brain images with a known realistic atrophy. Using these simulations as a gold standard, an evaluation of the performance of three popular atrophy estimation methods ("Structural Image Evaluation, using Normalization, of Atrophy" (SIENA) [2], for cross-sectional studies (SIENAX) [3] and "Boundary Shift Integral" (BSI) [4]), on the basis of their robustness to bias field inhomogeneity and noise is presented. Influence of brain lesion load on the atrophy measurements is also investigated.

\section{Proposed Approach for Atrophy Simulation}

Designing methods that simulate realistic atrophy is of great importance for the evaluation of atrophy measurement techniques, since it is a way of generating ground truth data. In the literature, several approaches have been proposed for simulating brain atrophy. In [5], Karacali et al. have proposed a Jacobian-based method where deformation fields are estimated in order to induce the desired volume variations in the regions of interest. An additional penalization term is also considered in order to prevent the corner Jacobians from being negative in order to ensure that the estimated deformation field preserves topology. However, the penalization term cannot rigorously guarantee topology preservation and restricts the simulation of large atrophies in one go. Pieperhoff et al. have recently presented a similar approach relying on "Local Volume Ratio (LVR)" [6] instead of the Jacobian. Unfortunately, none of these methods address the problem of enforcing skull invariance, which is a desirable property for the simulation of realistic brain atrophy. The bio-mechanical-based approach proposed by Camara et al. 7] relies on a bio-mechanical model for simulating brain tissue deformation, using a finite-element approach. Their framework also incorporates the skull invariance constraint.

Here, we present an alternative to the methods discussed above. The proposed approach estimates a deformation field that preserves topology so that the Jacobian is at each voxel, as close as possible to the desired local level of atrophy. Contrary to Karacali et al. [5] who consider the sum of squared differences between the Jacobian of the transformation and the desired level of atrophy, we consider the logarithm of the Jacobian so that dilations $(1<J<+\infty)$ and 
contractions $(0<J<1)$ have a similar influence on the objective function. Besides, additional constraints are introduced in order to ensure that the skull remains invariant by the estimated transformation. The proposed approach is detailed in the following section.

\subsection{Optimization Problem}

We consider a B-spline based multi-resolution deformable model (For details see [8]).Let $s \triangleq[x, y, z]^{t} \in \Omega \subset \mathbb{R}^{3}$. Let $\Omega_{J} \subset \Omega$ be the area where the desired simulated atrophy level $J(s)$ (the value of the Jacobian at each voxel $s \in \Omega_{J}$ ) is user-specified. For estimating the corresponding deformation field $\boldsymbol{u}$, we consider the following objective function:

$$
E \boldsymbol{u}, J, \lambda=\int_{\Omega_{J}}\left|\log \left(J_{\boldsymbol{u}}(\boldsymbol{s})\right)-\log (J(\boldsymbol{s}))\right|^{2} d \boldsymbol{s}+\lambda C \int_{\Omega} E_{R e g}(\boldsymbol{u}(\boldsymbol{s})) d \boldsymbol{s},
$$

where $J_{\boldsymbol{u}}$ stands for the Jacobian of $\boldsymbol{u}, E_{\text {reg }}$ is a regularization term that ensures that the estimated transformation is smooth, $\lambda$ is the weight of the regularization term and $C$ is a scaling factor computed at the beginning of each scale 9 . Among the many regularization terms proposed in the literature, we choose the membrane energy. We guarantee exact topology preservation by maintaining the positivity of the Jacobian in the continuous domain. As opposed to Karcali et. $a l$, who need an additional term for topology preservation, we directly solve the following constrained optimization problem.

$$
\hat{\mathbf{u}}=\arg \min _{0<J_{\mathbf{u}}(s)<+\infty} E \boldsymbol{u}, J, \lambda .
$$

The procedure for solving this optimization problem is quite involved and is detailed in 8 . We use the Levenberg-Marquardt optimization procedure in order to improve the convergence rate. In our framework, we invoke the skull invariance constraint by optimizing those B-spline parameters that do not affect the skull, while setting the other parameters to zero. Finally, to obtain the warped image, it is more convenient to consider the backward transformation so that standard interpolation techniques can be used for the regularly sampled data.

\section{Experimental Results}

\subsection{Simulation of Atrophy}

In this section, we study the performance of the proposed atrophy simulation algorithm. First, we investigate the influence of considering the logarithm of the Jacobian in the objective function ( $L o g$-norm) instead of the standard sum of squared differences $\left(L_{2}\right.$-norm). Fig. [1 highlights the fact that using the $L_{2}$ norm leads to nearly the same dispersion of Jacobian values, whatever be the simulated atrophy rate, whereas the Log-norm shows a constant relative dispersion, which is more consistent. A quantitative analysis of the ability of the proposed 


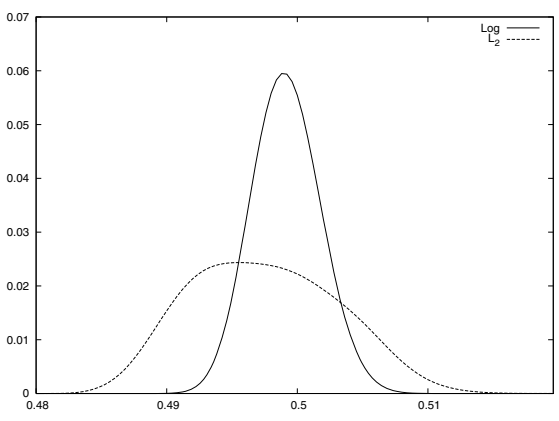

(a)

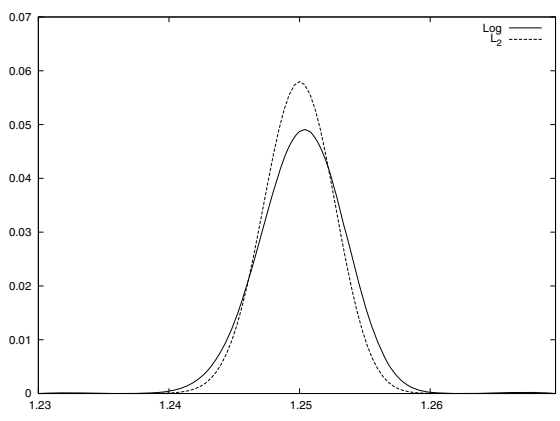

(b)

Fig. 1. Jacobian distributions obtained by simulating uniform (a) atrophy of $50 \%$ and (b) hypertrophy of $25 \%$

Table 1. Influence of considering the skull constraint on the mean and standard deviation of Jacobian values of the simulated deformation fields

\begin{tabular}{ccc}
\hline Desired Atrophy Without skull constraint With skull constraint \\
\hline $10 \%(\mathrm{~J}=0.9)$ & $0.9017 \pm 0.0021$ & $0.9032 \pm 0.0115$ \\
$20 \%(\mathrm{~J}=0.8)$ & $0.8015 \pm 0.0021$ & $0.8019 \pm 0.0202$ \\
$40 \%(\mathrm{~J}=0.6)$ & $0.6008 \pm 0.0018$ & $0.6025 \pm 0.0797$ \\
$50 \%(\mathrm{~J}=0.5)$ & $0.5005 \pm 0.0017$ & $0.5140 \pm 0.2088$ \\
\hline
\end{tabular}

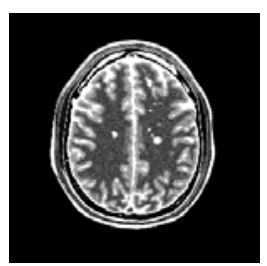

(a)

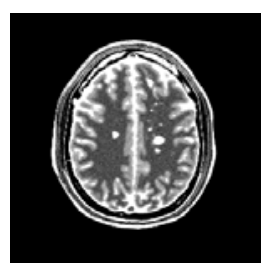

(b)

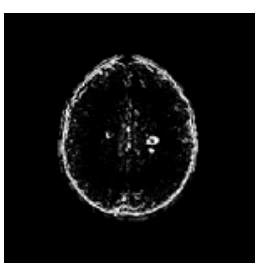

(c)

Fig. 2. Simulation of brain atrophy with an increase in MS lesion load: a) Original BrainWeb image with moderate lesions (b) Image with $10 \%$ of global atrophy and $100 \%$ of MS lesion increase (c) Difference between images (a) and (b)

algorithm for simulating the desired atrophy has also been performed (see Table 1). Simulations have been performed by considering a uniform atrophy over gray and white matter using the Brainweb 1 image. It can be seen that, on the average, the desired atrophy is well achieved without and with the skull constraint. Note that the proposed simulation algorithm can easily achieve very high atrophy, contrary to the method proposed in [5], which requires the estimation

\footnotetext{
1 http://www.bic.mni.mcgill.ca/brainweb/
} 
of a large atrophy in an incremental way. For example, it is possible to simulate a uniform atrophy of $99.9 \%(J=0.001)$, without the skull constraint, with an obtained average Jacobian value of $0.00106 \pm 0.000716$. Such an atrophy rate, although unrealistic, highlights the ability of the proposed method to produce an accurate solution for low Jacobian values. The proposed simulation framework is also versatile and can be used for simulating a more complicated pattern of atrophy. For instance, it can be used for simulating a global brain atrophy and a change in a given pathological area such as multiple sclerosis lesion evolution or tumor growth, simultaneously. In Fig. 2, we present a simulation of $10 \%$ of global brain atrophy and $100 \%$ of MS lesion volume increase using an image with "Moderate" lesion load from the BrainWeb MS database.

\subsection{Evaluation of SIENA, SIENAX and BSI}

In this paper, we use the SIENA and SIENAX implementations available as a part of the FMRIB Software Library (FSL) version 4.12. We use the BSI implementation developed by Imaging of Dementia and Ageing lab, University of California, Davis 3 . While the implementations of SIENA and SIENAX are completely automated, the implementation of BSI requires manual intervention for obtaining a gray-white matter mask, in order to define the brain boundaries on which the boundary shift integral is calculated. This problem is automatically alleviated in our case since the gray-white matter mask of the baseline image is available (with BrainWeb). We refer the reader to [2, 3] and [4 for a complete description of SIENA, SIENAX and BSI algorithms, respectively.

Our evaluation framework consists of the simulation of a number of atrophies (uniform over the brain) on a single normal brain image of BrainWeb. These simulations are a simplified version of the atrophy that occurs in reality but are adequate for the purpose of evaluation of the atrophy estimation approaches. The results are illustrated by simulating brain atrophy ranging between 0-1\% (step size $0.1 \%$ ) and 1-10\% (step size 1\%). Although, the brain atrophy range of $0-1 \%$ is more relevant to neuro-degenerative pathologies, we also present results for larger brain volume changes, in order to better assess the accuracy of these methods. To comprehend the effect of bias field inhomogeneity and noise, we create three sets of images. The baseline image as well as the atrophied images are degraded (a) using different intensity non-uniformity (INU) fields (20\% INU) available with the BrainWeb database (b) by adding Gaussian noise to all the brain scans such that a signal to noise ratio (SNR) of $15 \mathrm{~dB}$ is achieved (c) bias field inhomogeneity followed by noise using the same parameters as in (a) and (b). Fig. 3(a-d) shows the PBVC between any two brain scan pairs, $B 1$ and $B 2$, such that the simulated atrophy on brain $B 1$ is less than that of $B 2$ (for the simulated atrophy ranges of $0-1 \%$ and $1-10 \%)$. Fig. 3 compares the methods under consideration with respect to the ground truth, for (a) the noise-free case and for observations degraded with (b) bias field inhomogeneity (c) noise (d)

\footnotetext{
2 http://www.fmrib.ox.ac.uk/fsl/fsl/list.html

3 http://neuroscience.ucdavis.edu/idealab/software/index.php
} 


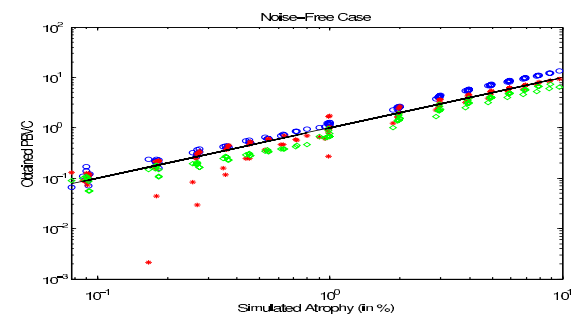

(a)

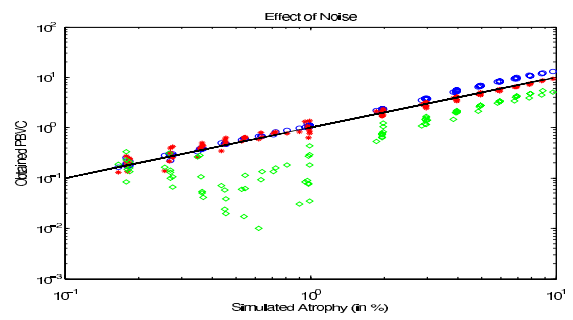

(c)

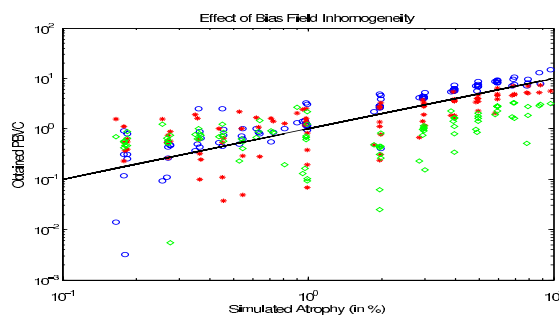

(b)

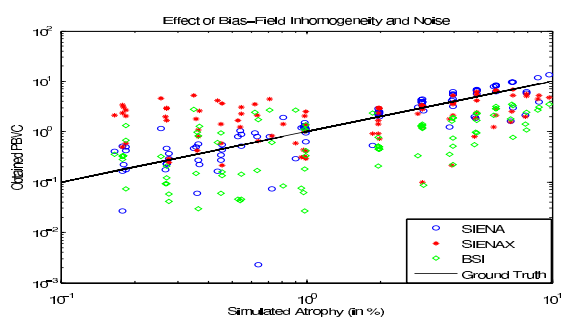

(d)

Fig. 3. Comparison of absolute errors in the estimated by SIENA, SIENAX and BSI with the ground truth for (a) noise-free observations and those degraded by (b) bias field inhomogeneity (c) noise (d) bias field inhomogeneity and noise

both bias field inhomogeneity and noise. In these figures, the absolute error in the estimated PBVC with reference to the ground truth is shown. Fig. [3(a) shows that SIENA overestimates the atrophy, while BSI underestimates it for large atrophy values when no artefact has been added. For low atrophy values (less than 1\%), SIENA, SIENAX and BSI show a similar performance in terms of the error in PBVC measured with respect to the ground truth. As can be seen from Fig. 3(d), the introduction of bias field inhomogeneity and noise leads to a visible increase in the errors for all the three methods. A prominent reason for this is the incorrect extraction of brain, when using BET, due to the addition of bias field inhomogeneity, mostly at the brain boundaries. In our observation, the addition of noise over bias field inhomogeneity leads to a significant change in the brain extraction as compared to the brain extraction performed when only bias field inhomogeneity is added (Fig. 3(b)). However, for the observations that are degraded with Gaussian noise only, we do not observe any gross errors in the extraction of the brain. Brain extraction is crucial for SIENAX because the segmentation performed on the brain area is directly related to the calculation of the brain volume. Although, SIENA uses a combined brain mask from the two examinations for the evaluation of the brain volume change, it can be seen that non-brain areas are included in the calculations if they are included in one of the examinations. This is the reason for the degradation of the performance of SIENA. In our experiments on BSI, a bias field correction, available with 
Table 2. Summary of results discussed in section 3.2 for the simulated atrophy range $0-1 \%$. This table illustrates the mean error in the estimated PBVC (in percentage) for various artefacts. Note that, for presence of lesions, the error represents the nondesired change that is observed when comparisons are done using different versions of the same brain with varying lesion load.

\begin{tabular}{c|ccc} 
Artefact & SIENA & SIENAX & BSI \\
\hline Noise-free & & & \\
Bias Field Inhomogeneity & $0.0615 \pm 0.0407$ & $0.0874 \pm 0.0759$ & $0.1072 \pm 0.0899$ \\
Noise & $0.2940 \pm 0.4343$ & $0.8420 \pm 0.7502$ & $1.0412 \pm 0.3827$ \\
Bias Field Inhomogeneity and Noise & $0.0292 \pm 0.0226$ & $0.0673 \pm 0.0433$ & $0.4400 \pm 0.1082$ \\
Presence of Lesions & $0.0941 \pm 0.0772$ & $0.2359 \pm 0.2089$ & -
\end{tabular}

the implementation of BSI, is applied after the registration step. Since the bias field correction step comes after the brain extraction and registration steps are performed, the error in these steps possibly propagate to the end. We note that, for BSI too, brain extraction is an important step since the final result depends on getting a good mask of the brain. Our experiments also suggest that an improper extraction of the boundary of the brain leads to a mis-calculation of the boundary shift integral. Also, the effect of addition of Gaussian noise only (Fig. 3(c)), does not have a significant effect on the measurements of SIENA and SIENAX but has an impact on the BSI measurements due to the use of intensity values directly in the calculation of the boundary shift integral.

To determine the effect of presence of lesions on atrophy, SIENA and SIENAX algorithms are run between "normal" brain and images with "mild", "moderate", "severe" lesion loads available with BrainWeb. In all these cases, no additional atrophy is simulated. Hence, it is expected that the atrophy estimated between these cases is close to zero. The deviations from zero atrophy represent the change in the estimated atrophy due to the presence of lesions. We observe that lesions can lead to a significant non-desired change of up to $0.2 \%$ when comparing a normal brain with the same brain with lesions using SIENA. SIENAX is more affected by the presence of lesions when a normal brain is compared with the same brain with lesions (maximum PBVC is $\sim 0.42 \%$ ). Since a gray-white matter mask delineating the brain boundaries is provided to BSI for carrying out its calculations, the presence of lesions is not expected to have any effect on atrophy estimation. Hence, BSI is not tested here. Table 2 summarizes the results corresponding to various sources of error discussed in this section. We would also like to bring to the knowledge of the reader that Smith et al. 10. report the overall mean absolute error of SIENA and BSI to be $\sim 0.2 \%$ which differs significantly from the results presented here (See Table 2). Smith et al. used incremental atrophy summation with first-last differencing for performing this evaluation [10]. Their calculation of mean absolute error is not based on a ground truth as is the case for our evaluation framework. 


\section{Conclusions}

In this paper, we proposed a topology preserving scheme for simulating atrophy using a B-spline based deformation model to create a gold standard for the comparison of atrophy estimation approaches. Additional constraints were introduced in order to ensure that the skull remains invariant in the simulated image and the estimated transformation is smooth. Our experiments showed that the proposed simulation algorithm can achieve the desired atrophy with accuracy. Using the simulated gold standard, we assessed the performance of three freely available algorithms (SIENA, SIENAX and BSI). Our analysis procedure consisted of simulation of atrophies on a single BrainWeb image in the presence of bias field inhomogeneity and noise. The experiments showed that, SIENA is the best performer with respect to the error in the estimated PBVC in the noise-free case as well as when the images are degraded with bias field inhomogeneity and noise. Bias field inhomogeneity and noise were responsible for incorrect brain extraction which considerably affected the accuracy of all the methods. Mean errors of $0.35 \% \pm 0.38,2.03 \% \pm 1.46$ and $0.91 \% \pm 0.80$ were observed in the estimated atrophy by SIENA, SIENAX and BSI, respectively. The observed errors were also significantly larger as compared to Smith et al. 10, who reported the overall mean absolute error for SIENA and BSI as $\sim 0.2 \%$. The tests that we performed also indicated that SIENA and BSI estimated longitudinal atrophy more accurately than SIENAX in a real scenario, where the images are corrupted with bias field inhomogeneity and noise. Since SIENAX has been developed for cross-sectional studies, its results should be cautiously interpreted when used in longitudinal studies. To conclude, the errors that we observed in our experiments were comparable to the whole brain annual atrophy rates $(0.5-2.8 \%)$ that have been reported for various pathologies. This highlights the need for the development of more robust methods capable of measuring atrophy accurately.

\section{References}

1. Simon, J.H.: Brain atrophy in multiple sclerosis: What we know and would like to know. Multiple sclerosis (Houndmills, Basingstoke, England) 12(6), 679-687 (2006)

2. Smith, S.M., De Stefano, N., Jenkinson, M., Matthews, P.M.: Normalized accurate measurement of longitudinal brain change. J. Comput. Assist. Tomogr. 25(3), 466-475 (2001)

3. Smith, S.M., Zhang, Y., Jenkinson, M., Chen, J., Matthews, P.M., Federico, A., De Stefano, N.: Accurate, robust and automated longitudinal and cross-sectional brain change analysis. Neuroimage 17(1), 479-489 (2002)

4. Freeborough, P.A., Fox, N.C.: The boundary shift integral: An accurate and robust measure of cerebral volume changes from registered repeat MRI. IEEE Trans. Med. Imaging 16, 623-629 (1997)

5. Karacali, B., Davatzikos, C.: Simulation of tissue atrophy using a topology preserving transformation model. IEEE Trans. Med. Imaging 25(5), 649-652 (2006) 
6. Pieperhoff, P., Sudmeyer, M., Homke, L., Zilles, K., Schnitzler, A., Amunts, K.: Detection of structural changes of the human brain in longitudinally acquired MR images by deformation field morphometry: Methodological analysis, validation and application. Neuroimage 43(2), 269-287 (2008)

7. Camara, O., Schweiger, M., Scahill, R., Crum, W., Sneller, B., Schnabel, J., Ridgway, G., Cash, D., Hill, D., Fox, N.: Phenomenological model of diffuse global and regional atrophy using finite-element methods. IEEE Trans. Med. Imaging 25(11), 1417-1430 (2006)

8. Noblet, V., Heinrich, C., Heitz, F., Armspach, J.P.: 3-D deformable image registration: a topology preservation scheme based on hierarchical deformation models and interval analysis optimization. IEEE Trans. Image Process 14(5), 553-566 (2005)

9. Noblet, V., Heinrich, C., Heitz, F., Armspach, J.P.: Retrospective evaluation of a topology preserving non-rigid registration method. Med. Image Anal. 10(3), 366384 (2006)

10. Smith, S.M., Rao, A., De Stefano, N., Jenkinson, M., Schott, J.M., Matthews, P.M., Fox, N.C.: Longitudinal and cross-sectional analysis of atrophy in Alzheimer's disease: Cross validation of BSI, SIENA and SIENAX. Neuroimage 36, 1200-1206 (2007) 